\title{
Assessing the dietary environment: examples from school-based nutrition interventions
}

\author{
Leslie A Lytle* and Jayne A Fulkerson \\ University of Minnesota, Division of Epidemiology, 1300 South 2nd Street, Suite 300, Minneapolis, MN 55454, \\ USA
}

\begin{abstract}
Objective: The objectives of this paper are to (1) introduce the concept of upstream and downstream public health approaches and discuss diet assessment issues in that context, and (2) provide examples of diet assessment methods and challenges in assessing environmental factors influencing eating patterns.

Design: Dietary assessment of environmental factors is discussed as they relate to nutrition interventions for school-aged children, although the issues transcend population characteristics. Examples of assessment challenges in measuring 'dietary environments' are drawn from the Child and Adolescent Trial for Cardiovascular Health (CATCH), Teens Eating for Energy and Nutrition at School (TEENS) and Trying Alternative Cafeteria Options in Schools (TACOS) studies.

Results/conclusions: The healthfulness of our 'dietary environments' (which may include food availability, social norms around food choice and the effect of pricing, policy and promotion on food choice) may be more important in determining what people consume than their individual decision-making about food choice. There is a dearth of published information to inform us on how to assess these 'dietary environments'.
\end{abstract}

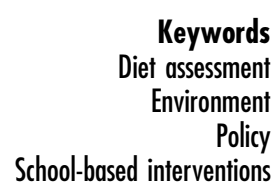

Keywords

Eet assessment

Policy

School-based interventions
Traditionally, diet assessment issues focus on obtaining valid and reliable information about individuals' or groups of individuals' food or nutrient intakes, addressing such questions as: What is the intra- and inter-individual variation in nutrients consumed over time? What assessment methods can we use to maximise the validity and reliability of self-reported intakes? What assessment tools work best in different populations? These are extremely important questions that will affect the quality of our nutrition surveillance data, our ability to evaluate the effectiveness of nutrition interventions and, more broadly, epidemiological evidence linking diet with morbidity and mortality of populations.

However, issues of diet assessment extend beyond the individual. The healthfulness of our 'dietary environments' (which may include food availability; social norms around food choice; and the effect of pricing, policy and promotion on food choice) may be more important in determining what people consume than their individual decision-making about food choice. There is a dearth of published information to inform us on how to assess these 'dietary environments'.

The objectives of this paper are to (1) introduce the concept of upstream and downstream public health approaches and discuss diet assessment issues in that context, and (2) provide examples of diet assessment methods and challenges at midstream and upstream levels. The issues are discussed as they relate to nutrition interventions for school-aged children, although the issues transcend population characteristics.

\section{The upstream/downstream paradigm}

The medical sociologist, John McKinlay, has popularised the notion of upstream and downstream approaches to public health interventions ${ }^{1}$. He recounts an allegory where an individual, standing downstream on the bank of a river, hears a cry for help from a drowning man and rushes into the water to save him. Just as one individual is saved, another individual cries for help and needs to be rescued. This Good Samaritan bystander is so busy saving individuals from drowning, he has no time to find out who is upstream pushing people in the river. The river becomes a metaphor for a continuum from system-level, public health approaches to individual-centred, medical model approaches to health ${ }^{2}$.

Downstream approaches to health occur primarily at the individual level or via a health professional working directly with a high-risk group. The medical model, where individuals come to the doctor for treatment or diagnosis, typifies downstream approaches. Downstream approaches deal primarily with tertiary prevention or minimising negative sequelae or further disability in those already inflicted with disease or a disabling condition. Typically, downstream interventions require active participation by individuals; they focus on volitional 
behaviour and expect individuals to seek out help and make decisions about changing their behaviour. Downstream approaches require the most 'work' from the individual.

Midstream approaches occur primarily at the group level. These groups may be healthy individuals who are at risk due to some level of risk exposure, and might include specific at-risk groups in communities, workers at a work site or even children in schools. In contrast, upstream approaches target the largest group with the least amount of discrimination. Efforts focus on primary prevention with the goal of eliminating or reducing risk exposure and keeping healthy people healthy. Upstream approaches are more passive interventions, requiring the least amount of 'work' or effort from the individual. The decision to change is made by someone else in authority or through legislation.

McKinlay and Marceau ${ }^{1}$ speak eloquently about the need for more upstream and midstream approaches to treating the health problems of the new millennium. Our biomedical and individual-centred treatment modalities are ill-equipped to solve complex problems such as obesity and cancer, where the environment is believed to contribute immensely to our behavioural choices and, ultimately, morbidity and mortality ${ }^{3}$. Even the new hope found in the mapping of the human genome is tempered by the realisation that our environment has more to do with our susceptibility to disease than does our DNA ${ }^{4}$.

\section{Diet assessment and the upstream/downstream paradigm}

Traditionally, our diet assessment methods have focused on downstream issues: 'What foods and nutrients are being consumed?' and, even more downstream, 'How do we assess the bioavailability of nutrients consumed?' Certainly, work has been done on midstream issues. Home shelf inventories ${ }^{5,6}$, grocery store shelf inventories ${ }^{7}$ and formative assessment of food availability in the community $^{8}$ are examples of assessing the availability of food - a midstream dietary environment issue.

Still, as our nutrition interventions expand to target the 'dietary environment', more attention must be focused on global issues that affect the dietary environment. For example, the state of the science for school-based nutrition interventions includes not only classroom curricula (with the goal of educating and motivating students to make healthful choices) but also modifying the school cafeteria or attempting to effect change in the child's food environment at home ${ }^{9-13}$. Recently, there has been a push to intervene at the policy level (the most upstream approach) through the use of taxation of soft drinks and snack foods to promote health ${ }^{14}$. French et al. ${ }^{15}$ suggest price manipulations as a way to increase the sales of more healthful items.

As our intervention focus changes, our measures for assessing the impact of our interventions change as well. Figure 1 illustrates a possible continuum of downstream, midstream and upstream approaches for reducing the intake of saturated fat and related diet assessment questions along the stream.

For intervention work as well as aetiological studies, there are benefits to assessing at multiple levels of influence ${ }^{16}$. System-level changes may be more sensitive to early programme effects than are individual-level changes. Therefore, documenting change in availability, promotion or pricing can serve as an early marker of intervention or mediating influences of behaviour change. In addition, system-level measures might be less biased than self-report of personal behaviour. Finally, it may be

\begin{tabular}{|c|c|c|}
\hline $\begin{array}{l}\text { Upstream-downstream } \\
\text { continuum }\end{array}$ & Treatment approach & Diet assessment questions \\
\hline & - Tax foods high in saturated fat & $\begin{array}{l}\text { How do we evaluate the effect } \\
\text { of taxation on sales and } \\
\text { individual consumption? }\end{array}$ \\
\hline Upstream & $\begin{array}{l}\text { - Create price incentives for } \\
\text { purchasing foods lower in } \\
\text { saturated fat }\end{array}$ & - How do we document sales? \\
\hline \multirow[t]{2}{*}{ Midstream } & $\begin{array}{l}\text { - Increase the availability of foods } \\
\text { low in saturated fat }\end{array}$ & $\begin{array}{l}\text { - What is the saturated fat } \\
\text { content of foods offered at } \\
\text { home, school, worksite? }\end{array}$ \\
\hline & $\begin{array}{l}\text { - Modify recipes to reduce } \\
\text { saturated fat consumption }\end{array}$ & $\begin{array}{l}\text { - How does recipe modification } \\
\text { affect consumption? }\end{array}$ \\
\hline Downstream & $\begin{array}{l}\text { - Reduce individuals' intake of } \\
\text { saturated fat }\end{array}$ & $\begin{array}{l}\text { What is the source and amount } \\
\text { of saturated fat in the } \\
\text { individual's diet? }\end{array}$ \\
\hline
\end{tabular}

Fig. 1 A possible continuum of downstream, midstream and upstream approaches for reducing the intake of saturated fat and related diet assessment questions along the stream 
more efficient and less costly to measure diet factors at the group, community or organisation level compared with individual-level assessments ${ }^{3}$. However, shifting diet assessment questions upstream brings new questions and challenges as well.

\section{Challenges in assessing the dietary environment}

To date, midstream and upstream diet-related outcomes have primarily been relegated to the status of secondary outcomes or process evaluation. As a result, study design, power analysis, and the rigor and appropriateness of measures for environmental and policy factors have taken a back seat to primary outcome measures that tend to be assessed at the individual level. The remainder of this paper looks specifically at some challenges in study design and measurement when assessing more upstream indicators of the 'dietary environment'. Examples are drawn from a variety of school-based nutrition interventions, including the Child and Adolescent Trial for Cardiovascular Health (CATCH), Teens Eating for Energy and Nutrition at School (TEENS) and Trying Alternative Cafeteria Options in Schools (TACOS).

\section{Obtaining variance estimates for environmental- level variables: example from the CATCH Eat Smart intervention}

CATCH was a large, multi-centred, school-based group randomised trial designed to develop and test the effectiveness of classroom, school and family approaches to decrease cardiovascular risk factors in children ${ }^{10}$. CATCH's primary hypothesis was that students exposed to the CATCH intervention would demonstrate significant reductions in their serum cholesterol levels relative to students in the control condition. Therefore, the primary outcome was serum cholesterol. Other individual-level outcomes assessed included anthropometric measures, blood pressure, diet, physical activity and smoking behaviours, and psychosocial predictors.

A very important element of the CATCH dietary intervention was to influence positively the school environment with regard to foods offered and promoted in the school cafeteria (CATCH Eat Smart intervention) ${ }^{17}$. Changes in the amounts of fat, saturated fat and sodium in foods offered in the school cafeteria were secondary outcomes. In order to evaluate the secondary outcomes of the intervention component, the diet assessment working group of CATCH needed to (1) estimate how many days of menus and corresponding recipes would need to be collected so that intervention effects could be detected in statistical analysis, (2) design a valid method of collecting menus and recipes from the $96 \mathrm{CATCH}$ schools, and (3) maintain quality control for data collection and data entry across four field centres ${ }^{18}$. While all of these questions were formidable challenges, for this discussion we focus on the first challenge.
Calculations to determine study sample size require that some estimate of variance for the measure in question is available. For individual-level outcomes, those variance estimates are often available via published research or via dialogue with researchers collecting data from individuals. Determining variance estimates for environmental-level indicators is much more difficult. In $\mathrm{CATCH}$, our school sample size was fixed at 96 schools based on power calculations for detecting change in serum cholesterol at the individual level ${ }^{19}$. However, it was unclear how many days of menu and recipe collection would be needed per school to detect changes in nutrients offered in the main trial.

Without any published or unpublished data available (the School Nutrition Dietary Assessment Study ${ }^{20}$ was being conducted concurrently with $\mathrm{CATCH}$ and variance estimates were not available), we decided to collect 20 days of menu and recipe information for all $96 \mathrm{CATCH}$ schools (close to 2000 days of menus and recipes). Twenty days of data collection were decided upon based on the belief that many schools had a four-week menu cycle and, in order to get a good estimate of the variability in nutrients available via the school lunch, a complete menu cycle would need to be documented. Data collection was expensive in terms of research staff time spent interviewing cooks/managers for menus and recipes, response burden for the school food service staff participating in data collection, follow-up with manufacturers regarding nutrient content of products and vendor foods, and other data collection and cleaning tasks. Once baseline data were analysed, we were able to examine the variance across CATCH sites, schools, weeks and days and determine that five days of menu and recipe data per school would provide adequate power to detect change between treatment conditions. Therefore, for interim and follow-up assessment periods, five days of menus and recipes were collected.

In retrospect, we could have collected menu and recipe data from a smaller number of days during our pilot work and used a bootstrapping procedure to derive variance estimates of our outcome variables. Those estimates could have been used to determine the number of days' worth of recipe data needed to detect intervention effects in the main trial. Bootstrapping is a non-parametric method for estimating variability of a sample ${ }^{21}$, in this case the variability of nutrients from recipes. It generates multiple samples by re-sampling with replacement from the available data. Variation among the bootstrap samples estimates sample variability. If time had permitted between finalising the menu and recipe data collection protocol during the pilot and beginning baseline data collection, variance estimates could have been derived from this iterative procedure. However, the amount of time it took to design and validate procedures to collect food service data ${ }^{17}$ and 24-hour recall data from 
elementary school children ${ }^{22}$ exhausted our limited resources.

\section{Minimising the potential for contamination in environmental outcomes: example from the TEENS study}

The TEENS study was conducted in 16 schools in the Minneapolis/St. Paul seven-county metropolitan area of Minnesota, with the goal of developing and evaluating curriculum, school-wide and family programmes to increase young adolescents' intakes of fruits, vegetables and lower-fat foods to reduce their future risk of cancer ${ }^{23}$.

The primary hypothesis of TEENS was that students in the intervention schools would eat more fruits and vegetables, and have a lower intake of energy from total fat, than students in the control schools. Dietary intake in students was assessed with 24-hour recalls and a fruit and vegetable screener ${ }^{24}$.

The sample size for TEENS was based on expected variances in students' intakes of fruits, vegetables and energy from total fat using estimates published in the literature and available from our previous research. We calculated that conducting the research in 16 schools with at least 30 teens per grade cohort per school would allow us to detect a difference in intake between treatment conditions of at least 1.14 servings of fruits and vegetables and $1.9 \%$ energy from total fat.

While there was a classroom curriculum and a family component to the TEENS programme, an equally important element of the intervention was to impact the school environment. One of the goals of the TEENS school environment component was to increase fruits and vegetables offered and sold on the school lunch line; a secondary outcome was to affect positively the availability and selection of fruits and vegetables on the school lunch line. We had the experience of the 5-a-Day Power Plus Study ${ }^{11}$ to calculate that five days of data on fruit and vegetables offered and sold would give us adequate power to detect change in that aspect of our school-level intervention. And, in fact, our baseline data revealed we were adequately powered to detect differences in the amount of 0.48 servings of fruits and vegetables sold.

Increasing offerings of fruits and vegetables requires input and support from the Food Service Director at the school district level. Consequently, food service directors were invited to food service training and received information, assistance and encouragement to incorporate TEENS goals in their schools randomised to the intervention group. While working with the district-level food service director was required by the intervention, it posed a threat to the internal validity of the study by introducing a potential source of contamination across study treatment conditions. TEENS recruited and randomised schools, not school districts, and seven school districts participated in the study. While four of the 16 schools represented four unique school districts, 12 of the 16 schools came from three large school districts and, in those cases, some schools were assigned to the control group while other schools within the same school district were assigned to the intervention group. After randomisation, only three control schools were 'pure' - uncontaminated by not having a district-level food service director exposed to any TEENS training or information. At the end of TEENS, all schools showed significant increases in sales of fruits and vegetables with no differences between experimental conditions $^{25}$. These findings suggest a strong secular trend in the control groups, mitigating our ability to see effects from the TEENS intervention.

The study design problem that impacted this secondary outcome was not easily rectified. Recruiting from 16 different school districts to ensure that all schools were uniquely assigned to the control or intervention group would have compromised one of the objectives of the study, which was to test the intervention in a lowerincome population. At the time of recruitment, the preponderance of lower-income students was located in the Minneapolis and St. Paul school districts. Using only one school from each of those districts would have required the other 14 schools to be recruited from the suburban or out-state areas, resulting in a sample that was made up predominantly of middle- to upper-middleincome students.

The study design was determined by using an individual-level measure (dietary intake) as the outcome measure. If environmental change had been the primary outcome measure rather than individual-level change, more school districts and possibly more schools would have been required to avoid the contamination caused by schools within a district randomly assigned to both experimental conditions. The TACOS study avoided this problem by using school districts, rather than schools, as the unit of randomisation. The TACOS study is used to demonstrate the next challenge to address in this paper: developing valid measures of the 'dietary environment'.

\section{Developing reliable and valid assessment tools for environmental endpoints: examples from TACOS}

The objective of the TACOS study was to examine a multicomponent environmental intervention to promote lowerfat, à la carte food selections and thereby lower dietary fat intake among adolescents in secondary schools. The main study outcome measures are percentage sales of lower-fat, à la carte foods (school-level data) and dietary fat scores from a food frequency checklist (individual-level data aggregated to the school level). The TACOS intervention focused on (1) working with school food service staff to increase the availability of lower-fat, à la carte foods in school cafeterias, (2) working with student groups to initiate student-peer promotion of lower-fat foods, and (3) lowering prices on lower-fat foods in school cafeterias.

A challenge faced in the pilot phase of TACOS was determining a valid method for collecting sales data on à la 
carte food items. Formative assessment revealed that most schools used computer software to run the financial end of their food service. Although there were various software programs available, many schools used a software program called PCS (developed by PCS Revenue Control Systems, Inc. in New Jersey) whereby a master list of foods is catalogued into the software and schools can generate customised overlays for computer terminals (similar to cash registers). Cashiers push the overlay keys to record foods sold. For example, the computer terminal might be set up with keys that identify 'Fresh fruit', 'Cookie' or 'Chips'. The PCS system holds great promise as a system already in place in schools that may be used to monitor sales and, therefore, serve as data collection for research. In addition, the PCS system has the advantage of producing continuous sales records by school-level staff, allowing for a relatively inexpensive method of collecting a great deal of data. Some adaptation to each school's system was expected for the intervention study, such as the addition of several keys on the overlays in order to document sales of new lower-fat products introduced as part of the intervention or when lower-fat foods and higher-fat foods were originally rung up together. In addition, some training of school food service staff was anticipated to make sure that products were keyed correctly and used consistently.

Pilot work revealed some additional, unanticipated challenges. It became apparent that terminal keys were often used to represent a price rather than an actual food item. For example, data under the 'Chips' key might actually represent a variety of items that all cost 35 cents. It was more important to school food service staff that students were charged the right price than whether the sales of individual items were reflected accurately in the sales reports. Therefore, TACOS evaluation staff needed to review carefully the products that were being sold to students and to determine if the products sold were being entered correctly into the software system by the cashiers. Another challenge was that new products were introduced frequently throughout the intervention period and a method was needed to update the study investigators about the new products. Repeated training proved to be essential for both intervention and control schools and included the following: why it is important to the study to have accurate keying; the study's definition of 'lower-fat'; and guidance on where to key food products, particularly new lower-fat items. Study investigators had to balance the risk of over-sensitising food service staff in control schools to the issue of fat content of products with the need to maximise the quality of data collected.

In response to the challenges encountered in the pilot work, protocols were instituted for the following: detailed, hands-on training; a monthly fax system for schools to send TACOS staff information on new products added to the à la carte lines; additional training for the cook manager/ head cashier for sending sales data files electronically from their school's computer; and continuous checks and balances between the sales reports and actual offerings and sales to students. One method for checking the accuracy of the sales data files includes a validation check in which a TACOS evaluation staff member observes and documents sales from one computer terminal line during one lunch period and compares his/her 'observations of sales' with a time-limited PCS sales report. These checks are conducted approximately every three weeks, and when the concordance between what the food service cashier enters (i.e. sales report figures) and what the evaluation staff documents is within $90 \%$ over the course of a three-month interval, the food service team receives a cash incentive.

\section{Using the appropriate data collection method}

The final example presented is drawn from the TEENS study and highlights the need for use of the most appropriate data collection methods to assess mid- and upstream approaches. One of the goals of the TEENS school environment intervention was to help schools establish school-wide policies to increase the availability and promote the choice of more healthful food and beverage selections in the school. To that end, School Nutrition Advisory Councils (SNACs) were established to help the stakeholders in schools make decisions and enact policies $^{26}$.

Detecting change in school policy was to be accomplished only through process evaluation, with no plans of making any statistical comparisons between treatment conditions. Still, we wanted to assess baseline and followup indicators of the presence of food policy in the schools as well as level of adherence to policies. At baseline, we conducted key informant interviews with school principals asking them if their school had a written food policy, to describe the written policy and any unwritten policies, and then to provide us with a copy of the written policy.

Twelve of the 16 principals said that they had a written school food policy but most seemed uncertain about any unwritten school policies. The majority of written school food policies dealt with student behaviour in the cafeteria, payment options for meals and the time available for meals in the school day. Policies that related to the nutritional content of foods available in the school simply documented that their breakfast and lunch programmes followed the guidelines set forth by the US Department of Agriculture for reimbursable meals.

Had we relied on asking a close-ended, quantitative question 'Do you have a school food policy?' we would have obtained a response that did not reflect the veracity of the situation. It was only through qualitative methods of interviewing individuals and listening to SNAC members talk about their perceptions of the school food environment that we were able to realise that most school stakeholders really did not know what we meant by a 'school food policy'. That lesson led us to do more 
formative evaluation with school stakeholders including surveys with parents and teachers where we asked about their perceptions of specific elements of the school food environment (i.e. food used as fundraisers, competitive foods in the school environment, vending and à la carte offerings).

The four examples presented illustrate only some of the challenges encountered when attempting to assess the 'dietary environment'. These are: (1) a lack of information on the variance of environmental-level outcomes to inform study size calculations; (2) study designs that cannot adequately ensure internal validity for both individual- and environmental-level outcomes; (3) the difficulty in collecting valid and reliable information on the 'dietary environment'; and (4) expanding our methods of data collection to include more qualitative methods. Many other challenges exist ${ }^{27}$.

\section{Conclusions}

As the limitations of our individually focused interventions (or downstream approaches) become more apparent, our nutrition interventions will focus more on affecting the dietary environments that support, influence and reinforce food choice $3,27,28$. That refocus brings with it more and unique challenges in assessing the dietary environment including, but not limited to, those illustrated in this paper.

The current state of research funding poses a fundamental challenge to using study designs appropriate for evaluating system-level outcomes. To date, the National Institutes of Health and other funding agencies that support large intervention trials have expected that outcomes are at the individual level rather than the environmental level. Since the rationale for health-related intervention trials is, ultimately, to decrease morbidity or mortality from disease, the traditional outcome is at the individual level since individuals - not environments get sick and die. Even in upstream intervention trials where the intervention emphasis is change in the environment and policy ${ }^{29,30}$, the primary outcome has been at the individual level and study design and power calculations so based. The TACOS study is an important exception to this trend and may mark the beginning of funding for primary outcomes at the environmental level. As long as the focus of intervention work is based on looking at changes at the individual level, rather than at the environmental, organisational policy or legislative level, much-needed work on developing and testing measures of the 'dietary environment' will be underfunded. We will continually be paddling upstream, battling a strong current.

One might argue that before funding agencies will be willing to devote research dollars to study change at midand upstream levels, we must be able to establish a causal link between the environment, individual choice and health outcomes. In other words, we will need to be able to prove that the environment is a mediating factor in health outcomes. Studying dietary environments as mediating factors brings with it challenges in assessing individuals' exposure to the environments in question. Unlike downstream approaches where we can document accurately who received nutrition counselling or attended a class to educate them on their dietary risk factors, quantifying individuals' exposure or reactions to manipulations in the 'dietary environment' will be much harder.

Finally, as we move forward in assessing mid- and upstream nutrition intervention approaches, our methods for data analysis must be appropriate. Data analysis methods that use the individual as the unit of analysis when it was actually the community, school or work site randomised to treatment condition, leads to overstatement of statistical significance by not taking into account the sameness that exists on outcome variables among individuals in a group ${ }^{1,16,31}$.

To date, our ability to assess mid- and upstream issues has been hampered by this simple fact: our research starts downstream and we battle to swim upstream to assess secondary outcomes that we know are important to address but are not funded adequately to measure. What the field needs is legitimisation by reviewers and funding agencies that 'putting in' midstream or upstream is an important and useful place to start. In the mean time, those of us swimming upstream can do our part by publishing and presenting mid- and upstream measures and outcomes and by working patiently to increase awareness of the value of mid- and upstream approaches in solving the complicated health problems faced by our nation and the world.

\section{References}

1 McKinlay JB, Marceau LD. Public health matters. To boldly go ... Am. J. Public Health 2000; 90: 25-33.

2 McKinlay JB. A case for refocusing upstream - the political economy of sickness. In: Enelow A, Henderson JB, eds. Applying Behavioral Science to Cardiovascular Risk. Houston, TX: American Heart Association, 1975.

3 Schmid TL, Pratt M, Howze E. Policy as intervention: environmental and policy approaches to the prevention of cardiovascular disease. Am. J. Public Health 1995; 85: 1207-11.

4 Lichtenstein P, Holm MV, Verkasalo PK, Iliadou A, Kaprio J, Koskenvuo M, et al. Environmental and heritable factors in the causation of cancer: analyses of cohorts of twins from Sweden, Denmark, and Finland. N. Engl. J. Med. 2000; 343: $78-85$.

5 Patterson RE, Kristal AR, Shannon J, Hunt JR, White E. Using a brief household food inventory as an environmental indicator of individual dietary practices. Am. J. Public Health 1997; 87: 272-5.

6 Crockett SJ, Potter JD, Wright MS, Bacheller A. Validation of a self-reported shelf inventory to measure food purchase behavior. J. Am. Diet. Assoc. 1992; 92: 694-7.

7 Cheadle A, Psaty BM, Curry S, Wagner E, Diehr P, Koepsell T, 
et al. Community-level comparisons between the grocery store environment and individual dietary practices. Prev. Med. 1991; 20: 250-61.

8 Gittelsohn J, Evans M, Story M, Davis SM, Metcalfe L, Helitzer DL, et al. Multisite formative assessment for the Pathways study to prevent obesity in American Indian schoolchildren. Am. J. Clin. Nutr. 1999; 69(Suppl.): 767S-72S.

9 Wechsler H, Deveraux RS, Davis M, Collins J. Using the school environment to promote physical activity and healthy eating. Prev. Med. 2000; 31: S121-37.

10 Luepker RV, Perry CL, McKinlay SM, Nader PR, Parcel GS, Stone EJ, et al. Outcomes of a field trial to improve children's dietary patterns and physical activity. The Child and Adolescent Trial for Cardiovascular Health. CATCH collaborative group. J. Am. Med. Assoc. 1996; 275: 768-76.

11 Perry CL, Bishop DB, Taylor G, Murray DM, Mays RW, Dudovitz BS, et al. Changing fruit and vegetable consumption among children: the 5-a-Day Power Plus program in St. Paul, Minnesota. Am. J. Public Health 1998; 88: 603-9.

12 Baranowski T, Davis M, Resnicow K, Baranowski J, Doyle C, Lin LS, et al. Gimme 5 fruit, juice and vegetables for fun and health: outcome evaluation. Health Educ. Behav. 2000; 27: 96-111.

13 Hearn MD, Baranowski T, Baranowski J, Doyle C, Smith M, Lin LS, et al. Environmental influences on dietary behavior among children: availability and accessibility of fruits and vegetables enable consumption. J. Health Educ. 1998; 29: $26-32$.

14 Jacobson MF, Brownell KD. Small taxes on soft drinks and snack foods to promote health. Am. J. Public Health 2000; 90: $854-7$.

15 French SA, Story M, Jeffery RW, Snyder P, Eisenberg M, Sidebottom A, et al. Pricing strategy to promote fruit and vegetable purchase in high school cafeterias. J. Am. Diet. Assoc. 1997; 97: 1008-10.

16 Diez-Roux AV. Bringing context back into epidemiology: variables and fallacies in multilevel analysis. Am. J. Public Health 1998; 88: 216-22.

17 Osganian SK, Ebzery MK, Montgomery DH, Nicklas TA, Evans MA, Mitchell PD, et al. Changes in the nutrient content of school lunches: results from the CATCH Eat Smart food service intervention. Prev. Med. 1996; 25: 400-12.

18 Ebzery ML, Montgomery DH, Evans MA, Hewes LV, Zive $\mathrm{MM}$, Reed DB, et al. School meal data collection and documentation methods in a multisite study. Sch. Foodserv. Res. Rev. 1996; 20: 69-77.

19 Zucker DM, Lakatos E, Webber LS, Murray DM, McKinlay SM, Feldman HA, et al. Statistical design of the Child and Adolescent Trial for Cardiovascular Health (CATCH): implications for cluster randomization. Control. Clin. Trials 1995; 16: 96-118.
20 Burghardt J, Gordon A, Chapman N, Gleason P, Fraker T. The School Nutrition Dietary Assessment Study: school food service, meals offered, and dietary intakes. Contract no. 533198-0-16. Sponsored by the US Department of Agriculture, Food and Nutrition Service, Office of Analysis and Evaluation. Princeton, NJ: Mathematica Policy Research, Inc., 1993.

21 Efron B. The Jackknife, the Bootstrap and Other Resampling Plans. Monograph 38. Philadelphia, PA: The Society for Industrial and Applied Mathematics, 1982.

22 Lytle LA, Nichaman MZ, Obarzanek E, Glovsky E, Montgomery D, Nicklas T, et al. Validation of 24-hour recalls assisted by food records in third-grade children. The CATCH Collaborative Group. J. Am. Diet. Assoc. 1993; 93: 1431-6.

23 Lytle LA, Perry CL. Applying research and theory in program planning: an example from a nutrition education intervention. Health Promot. Pract. 2001; 2: 68-80.

24 Murray DM, Phillips GA, Birnbaum AS, Lytle LA. Intraclass correlation for measures from a middle school nutrition intervention study: estimates, correlates and applications. Health Educ. Behav. 2001; 28: 666-79.

25 Lytle LA, Story M, Perry CL, Murray DM, Birnbaum AS, Kubik MY, et al. Can we effect change in adolescent fruit and vegetable intake? Paper presented at: American Public Health Association in Atlanta, GA, November 2001.

26 Kubik MY, Lytle LA, Story M. A practical, theory-based approach to establishing school nutrition advisory councils. J. Am. Diet. Assoc. 2001; 101: 223-8.

27 Richter KP, Harris KJ, Paine-Andrews A, Fawcett SB, Schmid TL, Lankenau BH, et al. Measuring the health environment for physical activity and nutrition among youth: a review of the literature and applications for community initiatives. Prev. Med. 2000; 31: S98-111.

28 Glanz K. Progress in dietary behavior change. Am.J. Health Promot. 1999; 14: 112-7.

29 Forster JL, Murray DM, Wolfson M, Blaine TM, Wagenaar AC, Hennrikus DJ. The effects of community policies to reduce youth access to tobacco. Am. J. Public Health 1998; 88: 1193-8.

30 Perry CL, Williams CL, Veblen-Mortenson S, Toomey TL, Komro KA, Anstine PS, et al. Project Northland: outcomes of a community-wide alcohol use prevention program during early adolescence. Am. J. Public Health 1996; 86: 956-65.

31 Koepsell TD, Wagner EH, Cheadle AC, Patrick DL, Martin DC, Diehr PH, et al. Selected methodological issues in evaluating community-based health promotion and disease prevention programs. Annu. Rev. Public Health 1992; 13: $31-57$. 